\title{
Anti-inflammatory Actions of Adjunctive Tetracyclines and Other Agents in Periodontitis and Associated Comorbidities
}

\author{
Aruni Tilakaratne ${ }^{1}$ and Mena Soory ${ }^{2, *}$ \\ ${ }^{1}$ Department of Oral Medicine and Periodontology, Faculty of Dental Science, University of Peradeniya, Sri-Lanka \\ ${ }^{2}$ Periodontology King's College London Dental Institute, Denmark Hill, London SE5 9RW, UK
}

\begin{abstract}
The non-antimicrobial properties of tetracyclines such as anti-inflammatory, proanabolic and anti-catabolic actions make them effective pharmaceuticals for the adjunctive management of chronic inflammatory diseases. An overexuberant inflammatory response to an antigenic trigger in periodontitis and other chronic inflammatory diseases could contribute to an autoimmune element in disease progression. Their adjunctive use in managing periodontitis could have beneficial effects in curbing excessive inflammatory loading from commonly associated comorbidities such as CHD, DM and arthritis. Actions of tetracyclines and their derivatives include interactions with MMPs, tissue inhibitors of MMPs, growth factors and cytokines. They affect the sequence of inflammation with implications on immunomodulation, cell proliferation and angiogenesis; these actions enhance their scope, in treating a range of disease entities. Non-antimicrobial chemically modified tetracyclines (CMTs) sustain their diverse actions in organ systems which include anti-inflammatory, anti-apoptotic, anti-proteolytic actions, inhibition of angiogenesis and tumor metastasis. A spectrum of biological actions in dermatitis, periodontitis, atherosclerosis, diabetes, arthritis, inflammatory bowel disease, malignancy and prevention of bone resorption is particularly relevant to minocycline. Experimental models of ischemia indicate their specific beneficial effects. Parallel molecules with similar functions, improved $\mathrm{Zn}$ binding and solubility have been developed for reducing excessive MMP activity. Curbing excessive MMP activity is particularly relevant to periodontitis, and comorbidities addressed here, where specificity is paramount. Unique actions of tetracyclines in a milieu of excessive inflammatory stimuli make them effective therapeutic adjuncts in the management of chronic inflammatory disorders. These beneficial actions of tetracyclines are relevant to the adjunctive management of periodontitis subjects presenting with commonly prevalent comorbidities addressed here.
\end{abstract}

Keyword: Arthritis, cardiometabolic disorders, non-antimicrobial actions of tetracyclines, periodontitis.

\section{INTRODUCTION}

Tetracyclines have non-antimicrobial anti-inflammatory, pro-anabolic and anti-catabolic properties which make them excellent candidate pharmaceuticals for simultaneous adjunctive treatment of periodontal and prevalent systemic comorbidities commonly seen in periodontitis subjects. Aspects of their non-antimicrobial anti-inflammatory actions of relevance in the adjunctive management of periodontitis and inflammatory loading from prevailing systemic diseases are addressed.

Literature searches from the last 15 years pertaining to the inflammatory pathogeneses of periodontitis and systemic comorbidities such as CHD, DM and arthritis were made using relevant key words. Searches utilized Medline, Embase, Pubmed and other sources for original papers, systematic and other reviews of the literature. Due to the heterogeneity of work covered, this review does not lend itself to a traditional systematic review; it attempts to address

*Address correspondence to this author at the Periodontology King's College London Dental Institute, Denmark Hill, London SE5 9RW, UK; Tel: 0044 (0)20 3299 3591; Fax: 0044 (0)20 3299 3826;

E-mail: mena.soory@kcl.ac.uk mechanisms involved in their actions and interactions which helps to clarify some inconsistencies across population groups based on inherent subject variations, variable disease parameters and outcome measures used in diverse studies. Systematic reviews and meta-analyses in the context of specific outcome measures, conducted in accordance with recommendations of the Cochrane Collaboration are included.

\section{INHIBITION OF MATRIX METALLOPROTEINASE (MMP) ACTIVITY BY TETRACYCLINES}

The discovery of MMP inhibition by tetracyclines by mechanisms unrelated to their antibiotic activity was a major breakthrough [1]; and the discovery of animal collagenase, the first of a series of MMPs, now recognized as essential mediators of collagen, connective tissue and bone destruction in several chronic inflammatory diseases including periodontitis. Collagenases break down connective tissue macromolecules and these include matrix metalloproteinases (MMPs). Tetracyclines inhibit MMP activity by depriving them of divalent cations which are co-factors for their activity. They also chelate these cations, thereby reducing their protein degrading activity. Chemically modified tetracyclines (CMT) are agents that have been developed to remove the anti-microbial actions of tetracyclines, while retaining 
their non-antimicrobial properties, by modifying the tetracyclic naphthacene carboxamide ring of the molecule. Tetracycline and its analogs are well known as a group of antimicrobials with a broad spectrum of activities. They act against a large number of gram positive and gram negative bacteria, including many putative periodontal pathogens. However, research carried out in the past reveals that tetracyclines possess other unique non-antimicrobial properties such as anticollagenase activity. These actions of tetracyclines are observed at sub-antimicrobial doses [2].

The above observations led to the development of subantimicrobial doxycycline dosing (SDD) and CMT formulations, for the treatment of periodontal disease, dermatological conditions; and as anti-angiogenesis agents as evidenced in human clinical trials, in certain types of cancer. Tetracyclines are able to inhibit both intracellular and extracellular MMPs. The active MMP-inhibitor site of the tetracycline molecule has been identified as the calcium and zinc binding site at C-11 and 12. As MMPs are required for physiological functions including matrix turnover, it would not be desirable to inhibit MMPs excessively. A pivotal multicentre randomized clinical trial in post-menopausal women with periodontitis and systemic osteopenia demonstrated that doxycycline at SDD could reduce progressive alveolar bone loss associated with periodontitis and systemic bone loss in the same subjects [3]. The efficacy and safety aspects of usage and future directions in managing oral and systemic bone loss are addressed. SDD was designed to suppress hostderived MMPs in the periodontal lesion in order to attenuate pathological degradation of various collagens including types 1,111 and $1 \mathrm{~V}$, while preserving other periodontal tissue components (fibronectin, proteoglycan ground substance, elastin and basement membranes). It inhibits bone resorption and minimizes side-effects of regular dosing.

CMTs can inhibit MMP-3 and serine proteinases, particularly neutrophil elastase. These properties confer connective tissue and bone sparing effects. MMPs can partially degrade and inactivate the endogenous serine proteinaseinhibitor $\alpha 1$-antitrypsin in serum and biological fluids. When MMP activity is blocked by tetracyclines, $\alpha 1$-antitrypsin is spared; enabling its inhibitory actions on pathologically elevated elastase activity. The binding energy calculated in solution predicts that CMT-3 complexes are the most favorable, followed by CMT-7 and CMT-8 analogs respectively; based on the mechanisms of action of CMTs as MMP inhibitors at a molecular level [4]. Photosensitivity is a reported side-effect of CMT-3, in a dose-responsive manner [5].

\section{APPLICATIONS OF ANTI-INFLAMMATORY AC- TIONS OF TETRACYCLINES IN INFLAMMATORY DISEASES}

There has been significant recent interest in the nonantimicrobial biological actions of tetracyclines with good coverage in the literature; which include anti-inflammatory, anti-apoptotic, anti-proteolytic actions, inhibition of angiogenesis and tumor metastasis [6]. The second generation semi-synthetic tetracycline, minocycline has been in therapeutic use for over 3 decades. Multiple non-antimicrobial biological actions in the context of inflammatory pathogene- ses seen in dermatitis, periodontitis, atherosclerosis, arthritis and inflammatory bowel disease; anti-proliferative actions in malignancy and prevention of bone resorption are especially relevant to minocycline. Several studies have been done on the neuroprotective actions of tetracyclines. Some side effects of minocycline associated with long-term usage for dermatological conditions include tooth discoloration [7]. This would not be applicable to conventional applications in the adjunctive management of periodontitis.

MMPs play an important role in the degradation of ECM resulting in cell migration, wound healing and remodeling of tissues. They constitute a group of more than $20 \mathrm{Zn}$ dependent enzymes crucial in ECM biology acting as regulators of inflammation in fine tuning acute and chronic inflammatory episodes; being key components in the progression of periodontitis. MMPs are essential for the turnover of ECM components. However when they are pathologically elevated, connective tissue and bone loss occur in a range of inflammatory diseases. Tetracyclines are effective inhibitors of mammalian MMPs. Sub-antimicrobial formulations of tetracyclines are effective in modulating the host response in periodontitis and dermatological conditions.

In view of similarities in the enolic system of curcumin and the C-11/C-12 diketonic moiety of tetracyclines in the inhibition of MMPs via Zn-binding; a uniquely modified core molecule of curcumin has been developed as a congener along these lines, in the context of its beneficial antiinflammatory effects in diseases associated with connective tissue loss [8]. The modified curcumin molecule has improved zinc binding and solubility; and significant actions in reducing excessive MMP levels and activity. The new curcuminoid bi- and tri-carbonylmethanes show substantial improvement in these properties. An N-phenylaminocarbonyl derivative of bis-demethoxycurcumin (CMC2.24) has been shown to be most effective in vitro amongst a series of neutral proteases implicated in connective tissue destruction. Oral administration of this compound to diabetic rats resulted in significant reduction in pathological levels of MMP-9 to normal values; without any toxic effects, demonstrated in cultured peritoneal macrophages. This novel compound could be effective in inflammatory diseases with connective tissue degradation such as periodontitis.

Selective inhibition of MMPs associated with disease progression would be a relevant therapeutic strategy, while the actions of MMPs affecting tissue turnover and detoxification need to be retained. Derivatives of monoclonal antibodies have potential therapeutic applications, particularly where specificity is paramount [9]. Increased target binding affinity of a monoclonal antibody confers high potency and selectivity linked to a drug scaffold with effective pharmacological properties. The second generation semi-synthetic tetracycline, minocycline has been used effectively over the decades. In addition to antimicrobial actions, it is also an effective anti-inflammatory and anti-apoptotic agent. Diverse experimental models of non-infectious diseases have demonstrated anti-proteolytic effects, suppression of angiogenesis and tumor metastasis. Several studies have addressed the non-antimicrobial actions of tetracyclines such as enzyme inhibition, antioxidant effects, immune cell activation and proliferation; and inhibition of apoptosis. These findings are 
reviewed, focusing on mechanisms underlying antiinflammatory and immunomodulatory actions of minocycline [10].

Most tetracyclines have the ability to inhibit MMPs. Their multiple mechanisms of action have been reviewed [11]. Minocycline crosses cell membranes readily and has potent anti-apoptotoic actions; doxycycline is an effective anti-protease and inhibits MMPs which contribute to tissue destruction in periodontitis and other inflammatory diseases. There is extensive documentation supporting the beneficial actions of tetracyclines, including their ability to scavenge ROS and act as anti-inflammatory agents. The pathogenesis of chronic inflammatory diseases results in increased formation of ROS and its sequelae leading to oxidative damage and dysfunction of cells. Tetracyclines have the capacity to scavenge free radicals and overcome oxidative stress [12].

They belong to the classic phenolic class of antioxidants, with antioxidant properties consistent with their structure; being similar to that of vitamin E. Minocycline demonstrates radical scavenging activity comparable to that of tocopherol, demonstrated in cell-free mixed-radical assays [13, 14]. It is effective as a superoxide scavenger, in quenching hydrogen peroxide [15] and peroxynitrite by direct interaction with free radicals. The diethylamino group on the phenolic carbon is unique to minocycline amongst tetracyclines, providing improved steric hindrance and confers superior radical scavenging activity on minocycline. It is $200-300$ fold more potent than tetracycline. It is also 200 -fold more potent than tetracycline in its inhibition of lipid peroxidation $[14,16]$. The mode of action of tetracyclines in a non-antimicrobial capacity addressed above indicates their potential adjunctive applications in periodontitis and prevalent comorbidities. Some examples of their actions in this context are addressed in the sections below.

\section{PERIODONTITIS AND TETRACYCLINES AS THERAPEUTIC ADJUNCTS}

Periodontal disease (PD) is a common inflammatory disease initiated by bacterial plaque biofilm, particularly in adult populations world-wide. Presentation of $\mathrm{PD}$ can range from 'gingivitis' which is the initial inflammatory condition affecting soft tissues surrounding the tooth, to the more advanced stage of the disease, periodontitis resulting in destruction of supporting structures of teeth. PD is initiated by bacterial plaque biofilm. Periodontal tissues respond to bacterial invasion by mobilizing defense cells and releasing inflammatory cytokines such as interleukins (IL) and tumor necrosis factor alpha (TNF- $\alpha$ ) which ultimately cause tissue destruction by stimulating the production of enzymes such as MMPs [17]. There is increasing evidence of the plausible association between PD and an increased risk of systemic diseases [18] such as diabetes mellitus (DM), atherosclerotic cardiovascular disease (ACVD), rheumatoid arthritis (RA) and adverse pregnancy outcomes. This article reviews their clinical and biological inter-relationship with these links; and treatment responses following therapeutic approaches with tetracyclines and other anti-inflammatory agents.

Periodontitis is the most prevalent chronic inflammatory condition in humans, resulting in destruction of supporting structures of teeth; occurring predominantly from an overexuberant host response to plaque biofilm. Current thinking indicates that successful long-term management of PD would combine local mechanical therapy directed against biofilm with host-modulatory strategies to overcome microbial and inflammatory burden [19]. Based on the nonantimicrobial, anti-inflammatory properties of tetracyclines, a host-modulatory agent comprising a subantimicrobial dose of doxycycline was developed (SDD), as a safe and effective adjunct to conventional non-surgical root surface debridement to treat chronic periodontitis. It has proved to be safe for use at $20 \mathrm{mg}$ b.d for at least 3 months and up to 24 months in randomized placebo controlled clinical trials. It is an approved adjunct in its capacity as an MMP inhibitor of plaque biofilm-induced degradation of tooth-supporting periodontal structures of the dentition. Doxycycline is preferred as a more potent inhibitor of MMPs than tetracyclines. It is safer than minocycline with superior pharmacokinetics which favor improved patient compliance.

Positive effects of intensive periodontal therapy extend beyond periodontal disease stabilization. Reduced inflammatory loading resulting from periodontal treatment could contribute to reduced risk of osteopenia, diabetes and coronary heart disease (CHD); considering the prevalence of common biochemical risk markers for these diseases based on the pathogenesis of chronic inflammation. Recognition of dual beneficial effects of periodontal host-modulatory therapy has resulted in the evolution of more effective CMTs, where the antimicrobial moiety is removed; retaining its potent actions in attenuating osteopenia and inhibiting MMP activity. Increasing recognition of the role of periodontitis in contributing to systemic comorbidities has led to the development of other host-modulatory agents such as resolvins [20] and PEZBINS (polyenolic Zinc-binding compounds), in an attempt to look for new molecules with the same active site as tetracyclines but with a different phenolic superstructure; they are in the preclinical stage of development [21]. In view of recent epidemiological studies that suggest underestimation of periodontal disease by $50 \%$ and increasing documentation of the link between PD and systemic comorbidities, this aspect of adjunctive therapeutics in periodontitis is likely to expand.

The adjunctive use of antibiotics is indicated in situations where root surface debridement alone has significant limitations. However they have side effects and their use should be limited to the more rapidly progressive forms of the disease. A meta-analysis of randomized controlled clinical trials demonstrates that supplementation of non-surgical periodontal therapy with systemic antimicrobials: amoxicillin with clavulanic acid and metronidazole; or subantimicrobial dosing with doxycycline; provides statistically significant results in subjects with rapidly progressive (aggressive) periodontitis with improved periodontal parameters of disease stabilization [22].

There are well-documented non-antimicrobial properties of tetracyclines such as minocycline in experimental models and in clinical trials in support of their evaluation as an effective adjunctive therapeutic approach for diseases with a dysregulated immune response. In diseases such as periodontitis with a microbial etiology and an altered immune patho- 
genesis, these compounds have particular relevance due to their anti-microbial and immunomodulatory effects. At conventionally detected serum and gingival crevicular fluid (GCF) levels, minocycline caused significant stimulation of osteoblastic cells, with a proportional increase in mineralized bone matrix in response to long-term exposure [23]; in the absence of effects on survival protein expression in human gingival fibroblasts, epithelial cells and periodontal ligament fibroblasts. In conjunction with their antimicrobial activity, these actions could explain the efficacy of tetracyclines, specially minocycline in decreasing disease progression and enhancing periodontal healing when administered for 7-14 days at 100-200 mg/day [24, 25] as an adjunctive measure.

In view of their importance in an inflammatory milieu, MMP inhibitors have evolved; they play a pivotal role in modulating chronic inflammation and enhancing repair and regeneration. Selective use of appropriate MMP-inhibitors as adjuncts could empower treatment outcome of periodontitis. Perioceutics, in the context of adjunctive therapeutic agents to complement root surface debridement for enhanced periodontal treatment outcome, potentially expand the scope for periodontal disease management. The actions of MMPs and their inhibitors in the pathogenesis of chronic inflammation; associated with enzyme cascades and perturbation of equilibrium with their natural inhibitors; and a role for the adjunctive use of MMP inhibitors in periodontal therapy have been reviewed. Current challenges and an overview of modalities achieved to date are addressed [26].

Clinical therapy directed at attenuating mediators of connective tissue breakdown is used in the adjunctive management of periodontitis [27]. Non-antimicrobial actions of specific tetracycline formulations cause inhibition of MMP-8 and MMP-13 [28]. Therapeutic actions of the tetracycline analog doxycycline hyclate include collagenase inhibition which makes it a useful therapeutic adjunct for the management of periodontitis [29] in modulating the host response, associated with an over-exuberant inflammatory response to plaque biofilm. Adjunctive sub-antimicrobial doxycycline as an adjunct to root surface debridement for stabilization of periodontal disease is a well-documented and established therapy. Clinical trials using this adjunct, demonstrate maintenance of alveolar bone over a six month period, when compared with placebo [30]. Increased periodontal disease severity, demonstrated more effective disease control in response to SDD [31]. Several studies demonstrate that tetracyclines have therapeutic potential to enhance bone mass by stimulating bone formation and inhibiting bone resorption; by virtue of their anti-inflammatory and anti-collagenolytic actions. Proanabolic and anticatabolic actions of tetracyclines are aided by osteoblast stimulation, MMP inhibition and attenuation of bone resorption. They reduce pathologically elevated levels of MMPs, pro-inflammatory cytokines and other inflammatory agents. Bone resorption is decreased as a result of combined anti-protease apoptotic effects on osteoblasts and osteoclasts respectively [32]. CMTs have good therapeutic potential in the adjunctive management of inflammatory periodontitis associated with connective tissue and bone destruction.

A systematic review has demonstrated the efficacy of MMP inhibition by SDD in the adjunctive treatment of adult periodontitis; with improved clinical outcome comprising clinical attachment gain and reduced probing pocket depths that prevailed for 9 months [33]. A more recent doublemasked, randomized, placebo-controlled, multicentre study of 266 subjects with periodontitis demonstrated that those treated with a modified release adjunctive SDD formulation taken daily, displayed significant gain in clinical attachment level and probing depth reduction in response to periodontal intervention; when compared with those receiving periodontal treatment only [34].

Other therapeutic approaches include a proof-of-principle study using SDD as an adjunct in the augmentation of periodontal wound repair using access flap surgery in subjects with severe periodontitis, and did not qualify for regenerative therapy; in order to improve the biological response with attenuated MMP expression. This approach demonstrated improved periodontal parameters (clinical attachment levels: CAL and probing pocket depths: PPD), when compared with access flap surgery alone [35]. The adjunctive formulation reduced post-surgical bleeding on probing, probing depths and periodontal bone resorption during its administration; however there was no change in the periodontal microflora, beyond the effect of surgical intervention. MMP inhibitors are effective adjuncts in the management of periodontitis subjects with decreased bone mass; SDD aids attenuation of periodontal disease progression while maintaining bone mass $[36,37]$; during adjunctive SDD, collagen telopeptide fragments in gingival crevicular fluid are shown to decrease [38, 39]. Evidence suggests that inhibition of MMPs in periodontitis subjects is significantly effective in controlling disease progression when used as an adjunct to mechanical debridement, also relevant to peri-implantitis and those requiring surgical intervention.

Long-term use of MMP inhibitors in chronic inflammatory diseases needs to be considered with care in view of its downstream effects in controlling cytokine processing; particularly MMP8 and its decreased levels over a period of time. More selective MMP inhibitors targeting periodontitis and combination therapy with antimicrobial and hostmodulatory targets could overcome potential side-effects. Increasing interest in the role of MMPs in bone homeostasis in health and disease, improves clarity on its mechanisms of action. These aspects of host modulatory therapeutics have enhanced the prospects for adjunctive management of periodontitis; further work along these lines could contribute to a more focused therapeutic targeting. Doxycycline hyclate at 1-2 $\mathrm{mg} / \mathrm{kg}$ was established for SDD in Beagle dogs. There was significant improvement in the degree of periodontal attachment and bleeding on probing in response to SDD of $2 \mathrm{mg} / \mathrm{kg}$ compared with control dogs and dogs with SDD of $1 \mathrm{mg} / \mathrm{kg}$ [40]. This appears to be a suitable SDD in the model tested with implications for long-term management of inflammatory disorders such as PD.

The efficacy of SDD as an adjunct to root surface debridement was evaluated in periodontitis subjects [41]. The systematic review and meta-analysis were conducted in accordance with the Quality of Reporting of Meta-Analyses statement and recommendations of the Cochrane Collaboration. The quality of methodology used was determined via a Consolidated Standards of Reporting Trials- based assess- 
ment. In three randomized placebo-controlled clinical trials with similar SDD dosing (20 mg b.d, for 3 months) and posttreatment follow-up period ( 9 months), significant differences were observed for all clinical parameters tested (CAL, PPD, plaque and gingival indices, GCF analysis); in favor of root surface debridement with adjunctive SDD. Within its limitations, this meta-analysis supported long-term efficacy of adjunctive SDD therapy using the above periodontal parameters.

\section{TETRACYCLINES AND SYSTEMIC COMORBID- ITIES}

Non-antimicrobial actions of tetracyclines such as MMP inhibition play an important role in periodontitis and other systemic diseases. These actions include regulation of cytokines, antioxidant effects and modulation of leucocyte chemotaxis and activation [42]. These applications have been utilized over the decades in dermatology particularly for their anti-inflammatory actions. Removal of the antimicrobial moiety and retaining the actions indicated above, impart therapeutic efficacy on CMTs with applications for dermatological and other chronic inflammatory diseases including periodontitis.

The broad-spectrum antibiotic tetracycline acts at the ribosomal level where it interferes with protein synthesis. Its early applications in dermatology were followed by discovery of diverse mechanisms of action during inflammation, angiogenesis, apoptosis and proteolysis and bone metabolism. These non-antimicrobial properties of tetracyclines and their analogs in a range of diseases including periodontitis and autoimmune disorders such as rheumatoid arthritis have been reviewed, addressing their potential for appropriate clinical applications [43]. Induction of DM in a rat model resulted in universally raised levels of collagenase in any tissue; this could account for unusual periodontal breakdown associated with hyperglycemia and altered host responses resulting in the formation of advanced glycemic endproducts (AGE) and its squeal [44].

Increasing interest in minocycline as an antiinflammatory agent has led to the evaluation of its therapeutic efficacy in several experimental disease models such as inflammatory bowel disease [45], diabetes [46] and cardiac ischemia [47]. Some of the mechanisms involved in the antiinflammatory, immunomodulatory and neuroprotective effects of minocycline are due to inhibitory effects on key enzyme systems, enhancement of Bcl-2-derived effects resulting in protection against apoptosis [48] and inhibition of poly [ADP-ribose] polymerase1 (PARP-1) activity [49].

Minocycline blocks LPS-stimulated secretion of inflammatory cytokines and toll-like receptor (TLR)-2 surface expressions in microglial cells. It has been shown to attenuate mRNA expression of genes for IL-6, IL-1 , major histocompatibility complex (MHC) 11 and TLR-2 [50, 51]. The efficacy of minocycline as an MMP-inhibitor has been demonstrated in the treatment of neuroinflammation following experimental prothrombotic cortical ischemia [52]. It is an effective adjunct in the management of malignant gliomas, associated with a significantly attenuated expression of membrane type $1 \mathrm{MMP}$ in microglia associated with glioma [53]. Its anti-proliferative, anti-angiogenic and matrix- stimulatory activities enhance its potential as an anti-tumor anti-metastatic agent. Some of the mechanisms involved include induction of autophagic/caspase-3-mediated cell death in a tumor model of glioma cells [54].

The effects of minocycline in preventing and overcoming induced experimental colitis, thereby attenuating disease severity and decreasing mortality have been reported [55]. These effects are due to reduced iNOS and MMP expression in intestinal tissue. Intestinal anti-inflammatory effects of minocycline in experimental models of colitis have been confirmed by others [45]; it was more effective than metronidazole traditionally used for this purpose [56].

Beneficial effects of minocycline are related to its actions in modulating both immune and microbiological parameters. Minocycline has been shown to restore the imbalance in composition of intestinal flora, characteristic of experimental colitis, unlike other antibiotics. In a mouse model of reactivated colitis, the association of minocycline with a probiotic has been reported to be beneficial in these conditions [57]; by exerting a greater anti-inflammatory effect than individual agents and also attenuating the reactivation of colitis [58]. Multifactorial conditions such as inflammatory bowel disease benefit from the antimicrobial and immune modulatory actions of minocycline; it would have similar applications for the adjunctive management of periodontitis, also associated with a multifactorial etiopathogenesis.

\section{APPLICATIONS FOR TETRACYCLINES IN CORO- NARY HEART DISEASE (CHD)}

Minocycline is able to limit ischemic damage in the kidney, heart, lung and neural cells, both in vitro and in vivo [59-62]. In the context of disruption of the blood brain barrier following a stroke; resulting in increased ischemic injury caused by edema and hemorrhage, minocycline is effective in reducing infarct volume and defective neurological function in mice; by inhibiting microglial activation and significantly minimizing disruption of the blood brain barrier and hemorrhage [15]. Based on this preliminary evidence, oral administration of minocycline $(200 \mathrm{mg}$ ) for 5 days in stroke subjects with a therapeutic window of 6-24h after stroke onset, resulted in a better outcome than placebo [63]. Protective effects of minocycline against myocardial ischemia/reperfusion (I/R) injury have been attributed to inhibited expression of a protein, high mobility group box 1 (HMGB1), an early mediator of inflammation and cell damage in I/R injury $[64,65]$. Inhibition of poly [ADP-ribose] polymerase 1 (PARP-1) is also a possible cardioprotective function attributed to minocycline [66]. Under normal conditions PARP-1 activation promotes DNA repair. However, during I/R injury, excessive activation of PARP-1 leads to cell death. Minocycline has a protective effect on the survival of cardiac myocytes by inhibiting PARP-1 activity.

Minocycline has been shown to reduce plaque size and vascular stenosis in diet-induced atherosclerosis, via a PARP-1 and p27Kip1 (a cyclin-dependent kinase inhibitor, leading to cell cycle arrest)-dependent mechanism [67]. It has anti-proliferative effects resulting in reduced vascular smooth muscle cells (VSMC) in atherosclerotic plaques of high-fat diet mice treated with minocycline. In addition, it was established that its anti-proliferative effects on VSMC 
were dependent on $\mathrm{p} 27 \mathrm{Kip} 1$ which was expressed in vitro in both VSMCs and human aortic SMCs; and in atherosclerotic plaques analyzed ex vivo; p27Kip1 knock down abolished these effects. Minocycline also reduced PARP-1 expression especially in LDL-induced human aortic smooth muscle cells (SMCs). Reduced atherosclerotic plaque size mainly due to these actions of minocycline suggests a potential application in vascular complications. Clinical trials are required to confirm their applications.

Periodontitis is the commonest inflammatory disease affecting humans. There is increasing interest and recognition of its importance as a risk factor for atherosclerotic cardiovascular disease (ACVD). Non-antimicrobial tetracyclines are effective against inflammatory mediators including cytokines and MMPs associated with periodontitis and associated comorbidities with a chronic inflammatory pathogenesis. There is evidence of the efficacy of sub-antimicrobial doxycycline and CMT3 in reducing LPS/CRP-oxidized LDL complex-stimulated monocyte production of TNF- $\alpha$, IL-6 and MCP-1; relevant to the pathogenesis of periodontitis and ACVD [68]. One of the mechanisms involved in this inhibition involves suppression of the phosphorylation / activation of NFkB cell signaling pathway.

Preliminary data from clinical trials indicate that almost all acute coronary syndrome (ACS) subjects demonstrate moderate to severe periodontitis. This is a significantly greater incidence than in the general population. Other studies demonstrate that sub-antimicrobial doxycycline dosing (SDD) dramatically reduces serum hsCRP, IL-6 and MMP-9 levels of ACS subjects and significantly elevates cardioprotective HDL cholesterol and its core molecule apolipoprotein A-1, in subjects susceptible to ASCVD, presenting with periodontitis. A possible mechanism involves protection of apolipoprotein A-1 from proteinase activity by inhibition of MMP and preventing loss of HDL. These multiple effects of non-antimicrobial tetracyclines provide significant therapeutic potential for the treatment of chronic inflammatory diseases including periodontitis and its comorbidities such as ACVD. An association between chronic periodontitis and risk of ACS was studied in the context of serum PMN markers myeloperoxidase (MPO), MMP-8, tissue inhibitor of metalloproteinase (TIMP)-1 levels and MMP-8/TIMP-1 ratio [69]. Raised levels of markers and a high ratio of MMP vs. inhibitor are suggestive of increased risk of ACS.

MMP-8 belongs to the collagenase-2 subgroup of the MMP superfamily of calcium- and zinc- dependent neutral proteinases. MMP-8 is the most effective proteinase in causing degradation of type 1 collagen and extracellular matrix leading to loss of supporting structures in periodontitis and peri-implantitis leading to tooth/implant loss. MMP-8 in particular has been shown to be up-regulated in affected tissues, gingival crevicular fluid, peri-implant sulcular fluid and serum during the inflammatory pathogeneses of periodontitis, peri-implantitis and CHD. Pathologically excessive amounts of MMP-8 have been implicated in CHD, in the context of its proteolytic action contributing to thinning of the collagenous fibrous lining of coronary and other arteries leading to atherosclerotic plaque destabilization and rupture [70]. Serum levels of MMP- 8 have been proven to be sensitive and objective biomarkers of periodontitis, peri-implantitis and
CHD during disease progression and response to treatment with doxycycline. Serum and oral fluids are readily collected for such diagnostic and outcome applications; the relevance of MMP-8 to CHD makes it an important diagnostic tool. Adjunctive therapeutics with doxycycline for periodontitis resulting in its inhibition would also be a significant outcome for CHD.

Recent literature further provides substantial evidence for an apparent association between the presence of PD and a heightened risk of CHD. Although a causal relationship has not been established, due to difficulties in excluding other confounding epigenetic variables such as smoking and diverse life-style risk factors of CHD, it is possible to suggest a link between PD and CHD; both being chronic inflammatory diseases that affect a large number of individuals in a population. The suggested mechanisms which may play a role in linking PD and CHD have been described as 'direct bacterial effects', 'indirect inflammatory effects' and 'genetic predisposition' [71]. Subjects with PD may present with a large surface area of inflamed ulcerated pocket epithelium that interacts with microorganisms in the sub-gingival plaque biofilm which could gain access to the circulation and lead to vascular accidents in coronary vessels. $P$. gingivalis has been identified in coronary atheromatous plaques suggesting that, it could precipitate rupture of the fibrous membrane leading to either unstable angina or myocardial infarction (MI). In addition to a direct microbial influence, indirect inflammatory effects have been linked with the association between PD and CHD. PD induces an inflammatory response that elevates levels of acute phase proteins such as CRP and fibrinogen which are well established independent risk factors for CHD [72, 73]. Both CRP and fibrinogen are also linked to inflammatory periodontitis. In addition, other inflammatory cytokines produced during periodontal inflammation may also gain access to the circulation, resulting in proinflammatory effects at a distant organ such as the heart.

Considering the genetic predisposition for both PD and $\mathrm{CHD}$, it has been proposed that a hyper-inflammatory phenotype could be responsible for cellular and molecular links between PD and CHD. This hyper-inflammatory immune response could result in increased production of cytokines and other inflammatory mediators in response to LPSchallenge, promoting periodontal breakdown [72]. Similarly, inflammatory mediators such as IL- $1 \beta$, TNF- $\alpha$ and thromboxane- $\mathrm{A}_{2}\left(\mathrm{Tx}_{2}\right)$ could uphold platelet aggregation, formation of lipid-laden foam cells and deposition of cholesterol in the vascular intima [71]. It has been reported that the activity of monocytes could also be influenced by various lipid transport molecules, with low density lipoproteins (LDL) upregulating secretion of IL- $1 \beta, \mathrm{TNF}-\alpha$ and TxA $\mathrm{A}_{2}$.

Hypertensive patients suffering from MetS show increased oxidative stress and compromised anti-oxidant activity. In addition to this, obesity and being overweight are significantly linked to hypertension, while hyperglycemia and hypertension are also closely related. Hyperglycemia leads to excessive stimulation of the sympathetic nervous system which results in vasoconstriction, increased sodium reabsorption and subsequent water retention leading to increasing hypertension. This in turn would damage the vascular endothelium [74-76]. 
A relationship between obesity and PD has also been documented [77, 78]. Adipocytes of fat tissue demonstrate an ability to secrete adipokines which appear to control appetite and bodyweight of an individual. Since the cytokine leptin is known to have a protective role against obesity, the condition is also described as 'leptin-resistance' with consequent leptinemia $[79,77]$. Adipocytes can secrete other cytokines such as adiponectin and resistin. Under normal circumstances, the serum level of adiponectin remains constant, however it decreases in the presence of obesity, DM, insulin resistance and CHD. Resistin plays a significant proinflammatory role and appears to be associated with insulin resistance $[79,80]$.

It is evident that inflammatory loading from PD could give rise to multifaceted complications of CHD and DM in a systemically compromised individual [18]. High levels of glucose and fatty acids, not only lead to the production of reactive oxygen species (ROS), but the interaction between glucose and plasma proteins would result in the formation of advanced glycemic end products (AGE). AGE can initiate the synthesis of TNF- $\alpha$, IL-6, IL-1 $\beta$ and ROS, which would further prompt chemical modification of lipoproteins and atheromatous plaque formation [81]. Oxidative stressinduced pathogenesis of PD is considered to be similar to that occurring in the systemic inflammatory environment in response to systemic co-morbidities. In this context, there is a typical presentation of a cluster of risk factors in chronic MetS. Thus antioxidants such as tetracyclines are considered to be important adjuncts in the management of subjects with PD and the above-mentioned systemic inflammatory diseases [48].

Tetracyclines, especially doxycycline (DOX) with their inherent anti-oxidant properties have been known to be important inhibitors of MMPs; which proteolyze extracellular matrix proteins. MMP-2 in particular is considered to be an important intercellular protease which has the ability to proteolyze specific intracellular proteins in cardiac muscle cells to reduce their contractile function [82], thus contributing towards the pathogenesis of CHD. Therefore, inhibition of MMPs is a growing therapeutic aim in the treatment and prevention of CHD. A recent review further points out how MMPs can be intracellularly regulated and activated by oxidative stress, cleaving several vital proteins within the cells. These pertain to the intracellular actions of MMPs in CHD including ischemia, injury, inflammatory heart diseases and septic shock. These features underscore useful applications of tetracyclines as MMP inhibitors and their therapeutic value in treating patients with CHD.

Periodontitis which is a common chronic inflammatory disease in man has already been recognized as a possible risk factor for atherosclerotic cardiovascular disease (ASCVD). Non-antimicrobial tetracyclines have shown inhibitory effects on inflammatory mediators and effector molecules including cytokines and MMPs associated with both diseases. Research into mechanistic links between local and systemic inflammation shows evidence of doxycycline and related non-antibiotic chemically-modified tetracyclines (CMT-3), to be effective in reducing the production of TNF- $\alpha$, IL-6 and macrophage chemoattractant protein-1 (MCP-1) by human mononuclear inflammatory cells, when stimulated with either LPS endotoxin or by a complex of CRP/oxidized-LDL cholesterol relevant to the pathogenesis of PD and ASCVD respectively [46]. This study suggests that the inhibitory actions of tetracycline compounds are mediated partly by suppression of phosphorylation/activation of the NFkB-cellsignaling pathway. Clinical trials carried out on patients with PD and ASCVD reveal that subjects with acute coronary syndrome have a higher incidence of moderate to severe periodontitis than the normal population. Further, SDD demonstrates a dramatic reduction in hsCRP, IL-6 and MMP-9 levels in subjects with acute coronary syndrome.

A six-month course of SDD has resulted in favorable modifications in the inflammatory environment amongst subjects with active CHD [83]. This was the first human study to evaluate MMP inhibition with a tetracycline derivative in CHD subjects (Metalloproteinase Inhibition with subantimicrobial dose Doxycycline to prevent Acute coronary Syndromes: MIDAS). MIDAS was not powered to detect a difference in clinical endpoints; however at six months of SDD treatment there was a significant $46 \%$ reduction in hsCRP levels and a $32 \%$ reduction in IL-6 levels. It is relevant that the reduction in hs-CRP levels in response to SDD was seen amongst $>80 \%$ of subjects who were already on statins, also known to reduce hs-CRP [84]. These profoundly significant findings are supportive of a more central role for MMPs in addition to matrix degradation during inflammatory sequelae; it is also suggestive of an incremental effect of SDD on those achieved by statins, in reducing inflammation. Treatment with SDD has been shown to reduce the severity of chronic inflammation and increase serum HDL cholesterol. The ability of SDD to enhance serum-mediated cholesterol efflux from macrophages was found to be significant in a double blind randomized study of post-menopausal osteopenic women with periodontitis [85]. These findings suggest that treatment with SDD could reduce the risk of CHD in these periodontitis subjects, by increasing the cholesterol efflux capacity of serum.

SDD has also been found to significantly increase serum levels of HDL cholesterol (which is cardio-protective) and its core molecule apolipoprotein A-1 in ASCVD-prone patients with PD. This study suggests a possible mechanism that SDD could inhibit MMP-mediated HDL loss by protecting apolipoprotein A-1 from proteinase attack [68]; nonantimicrobial tetracyclines thus provide significant therapeutic potential for treating chronic inflammatory diseases such as PD and ACVD.

SDD permeates arterial walls to block endotoxin-induced secretion of TNF- $\alpha$ and IL-1 in cultured monocytes and down-regulates the expression and activity of MMP-2, MMP-9 and IL-6 [86, 87]. The ability of doxycycline to limit the expansion of abdominal aortic aneurysms can also be attributed to the inhibition of MMPs [88, 89].

Furthermore, IL-6 induces CRP production and its release was also reduced by SDD. This SDD-related reduction in high sensitivity CRP may result from the upstream inhibition of IL-6, a direct inhibitory effect on high sensitivity CRP synthesis or both [83]. The degree of inflammation as measured by hsCRP correlates with prognosis amongst sub- 
jects with established CHD as well as healthy individuals. It is not certain whether elevated levels of hsCRP could simply identify individuals with an increased risk of coronary events or actually cause these events by virtue of its pro-atherogenic properties. However, the prospect of a therapeutic agent with the ability to reduce CRP, IL-6 and MMP-9 has significant clinical implications.

MetS causes a pro-oxidant state in periodontal tissue, altering anti-oxidant defense mechanisms. This adversely affects tissue responses against bacterial plaque. Conversely, PD being a productive source of oxidative stress markers promotes the onset of insulin resistance and MetS in a vicious circle [90]. Chronic inflammation prevalent in PD causes increased neutrophil defense activity which involves increased oxidative activity resulting in peroxidation and oxidative stress. Thus, both MetS and PD show increased serum rates of oxidative stress markers [91]. While several studies have focused on molecules that can lower oxidative stress, recent studies have proposed tetracyclines as useful agents in counteracting oxidative stress in PD and MetS. Tetracyclines in conjunction with insulin have presented beneficial effects in collagen formation during wound healing. Topical tetracyclines have resulted in faster healing in diabetic animals, particularly during the initial phases of wound healing [92].

Decreased yields of oxidative stress markers have been observed in the presence of minocycline which demonstrates its potential role as an adjunctive therapeutic agent in an environment of oxidative stress such as PD and coexisting cardio-metabolic co-morbidities. While pharmacotherapeutic agents such as aspirin, statins, and thiazolidinediones appear to reduce inflammation [83], thymoquinone has demonstrated a variety of pharmacologic properties including antihistaminic, antihypertensive, hypoglycemic and antioxidative activities other than its anti-inflammatory and antibacterial actions. Thus, thymoquinone has also been proposed to have a key role in the prevention of inflammatory periodontal disease [72].

As addressed above, MetS is a syndrome characterized by several complications collectively leading to seriously compromised health of an individual. It is clear that the universal denominator underlying the MetS and PD is oxidative stress. The consequent hyper-inflammatory state leads to grave systemic complications such as $\mathrm{CHD}$ as well as local tissue destruction experienced in PD. Among other therapeutic agents tested in various studies, tetracyclines have convincingly demonstrated anti-oxidant, anti-inflammatory, proanabolic, immune-modulatory, angiogenic and anti-apoptotic properties [93], and therefore they can be effectively utilized to combat this central predicament. However, an enhanced collaboration between physicians, cardiologists, endocrinologists, dieticians and dentists would be important in the diagnosis and management of these patients to deliver both routine and adjunctive care.

\section{METS, DM AND APPLICATIONS FOR TETRACY- CLINES}

Metabolic syndrome (MetS) is considered to be a major systemic co-morbidity in the population world-wide. It is a cluster of multiple interlinked risk factors predisposing to atherosclerosis. These risk factors include obesity, dyslipidemia, hypertension, CHD and hyperglycemia. MetS has been identified as carrying a high risk for the development of type-2 DM [94]. Epidemiological reports suggest an association between PD and MetS, although the causality of these relationships has not been conclusive in some studies [9597]; inherent subject-based variation could account for some of these findings. However, a large population-based research study provides evidence of an association between MetS and severe PD [98]. Evidence of a positive association between obesity and PD has been reported in systematic reviews [99, 100]. Similar positive associations have been reported in prospective studies as well [101]. The antiinflammatory actions of tetracyclines would be effective in overcoming associated mechanisms of inflammation and oxidative stress resulting from cytokine cascades and their sequelae in MetS and periodontitis.

Increased plasma levels of cytokines such as IL-6 and TNF- $\alpha$ associated with hyperglycemia contribute to oxidative stress-induced cell damage [102]. Adipose tissue, a major source of the above cytokines is considered to be a 'lipid store' and an active endocrine organ. Adipose tissue thus secretes a number of hormones and mediators called 'adipokines'. In type 2-DM, there are elevated levels of circulating cytokines and adipokines. These may contribute to a heightened inflammatory state in periodontal tissues, contributing to increased PD in diabetes [103].

Administration of the anti-oxidant, glutathione completely suppressed elevation of cytokines in response to glucose pulses, implying a possible oxidative stress-induced mechanism responsible for the inflammatory burden due to hyperglycemia [104]. These actions could have implications on atherosclerosis and CHD [18]. In the hyperglycemic state, the interaction between glucose and structural proteins leads to non-enzymatic glycosylation, producing advanced glycation end products (AGE). AGE products could initiate the production of TNF- $\alpha$, IL- 6 , IL-18 and reactive oxygen species (ROS) which would contribute to chemical modification of lipoproteins and atherogenesis in diabetics [81]. Some of the oxidant effects of AGE are overcome by the antioxidant glutathione [105], suggestive of a role for tetracyclines as therapeutic adjuncts in this context. It is relevant that in cultured osteoblasts, decreased yields of anti-oxidant markers in response to bacterial lipopolysaccharide, glucose and glucose-oxidized low-density lipoprotein were overcome in response to minocycline [106]; thus reinforcing its potential actions as an antioxidant in an environment of oxidative stress. Extrapolation of the findings from this simulated model could be applied to PD and coexisting cardiometabolic risk markers in the context of its antioxidant actions relevant to healing.

The pathophysiology of DM and CHD / MetS has implications on the progression of PD. The common risk factor, oxidative stress-induced tissue damage is responsible for progression of the above systemic diseases as well as PD $[107,108,18]$. These factors underscore the applications of tetracyclines as therapeutic adjuncts in the management of the above disease entities.

Metabolic syndrome (Mets) comprises a cluster of cardiovascular risk factors including obesity, impaired glucose 
tolerance, hyperinsulinemia, hypertension and dyslipidemia. There is increasing documentation of the link between periodontitis and systemic comorbidities, associated with inflammatory loading and common risk markers of disease progression. Equilibrium between the production and inactivation of ROS is disrupted during oxidative stress which characterizes MetS. ROS are essential for physiological functions, but excessive production during oxidative stress could contribute to cellular damage and dysfunction. It could be a common link in the pathogenesis of MetS and periodontitis, resulting in a proinflammatory profile of risk markers of disease [72]. Adipocytokines released by fat metabolism in adipose tissue could modulate oxidant/antioxidant homeostasis, by virtue of their actions. Insulin resistance could result from elevated production of ROS due to increased calorific intake and raised metabolic activity. Greater insulin levels required to maintain glucose homeostasis in obese subjects could contribute to hyperinsulinemia which could then progress to DM. Increased neutrophil adhesion and chemotaxis in response to oxidative products could cause further oxidative damage. Oxidative stress and hyperglycemia could result in the formation of advanced glycation end products (AGE) which are implicated in damage to periodontal tissue. Inter-related mechanisms of oxidative stress in MetS could contribute to common risk factors arising from a chronic state of oxidative stress in the pathogeneses of periodontitis, CHD and DM.

There is a possible role for the inhibition of non-enzymic protein glycation by tetracyclines, suggested by in vitro and animal studies. In diabetic subjects on stable medication with oral hypoglycemics/insulin and untreated periodontitis, conventional subgingival debridement was compared with additional adjunctive therapy of antimicrobial doxycycline for 2 weeks/subantimicrobial doxycycline (SDD) for 3 months. At one and three months, there were significant improvements in $\mathrm{HbA} 1 \mathrm{c}$ in response to SDD compared with antimicrobial doxycycline or placebo [109]. These findings indicate that SDD overcomes oxidative stress via host modulatory mechanisms with decreased $\mathrm{HbAlc}$ values in type $2 \mathrm{DM}$ subjects on prescribed medication. Adjunctive SDD in periodontal therapy suppresses pro-inflammatory cytokines and regulates the inflammatory response to therapy [110]; this could contribute to the above mechanisms. GCF cytokine profiles in subjects with or without type $2 \mathrm{DM}$ seem to be governed by the intensity of periodontal inflammation and the role of DM could be a secondary factor in this context. In a literature review of ten comparable studies, 2 showed GCF IL-6 to be greater in periodontitis $+\mathrm{DM}$; while 3 demonstrated IL-1; 1 showed IL-17 and IL-23 and 1 indicated IL-8 to be elevated in GCF of subjects with periodontitis +DM over controls [111]. It is relevant that a poor correlation between GCF cytokine profiles in periodontitis in the presence or absence of DM may be indicative of the need for concurrent serum samples [112].

CMT suppress cataract formation in a diabetic rat model, partly by affecting MMPs. They may have a role in treating pivotal causes of vision loss [113]. Age-related macular degeneration and diabetic retinopathy are likely to increase due to an aging population. In the context of retinal disease, retinal cell apoptosis could be prevented by tetracyclines; in addition to prevention of retinal capillary damage via caspase inhibition; and prevention of retinal neovacularization by inhibition of MMP-2 and MMP-9. Other mechanisms include elevation of pigment epithelial derived growth factor and reduction in vascular endothelial growth factor (VEGF) expression via Fas ligand. Some of these actions could be relevant to the management of periodontitis subjects with DM.

\section{PERIODONTITIS AND RA}

While PD is caused by pathogenic bacteria such as $A g$ gregatibacter actinomycetemcomitans $(A a)$, Porphyromonas gingivalis (Pg), Bacteroides forsythus (Bf), Prevotella intermedia and Prevotella melaninogenica, these pathogenspecific immunoglobulins (IgG and $\operatorname{IgA}$ ) against some of these organisms have been found to be in the synovial fluid of patients with RA [114]. Among other periodontal pathogens, $P g$ is known to possess a unique microbial enzyme called, peptidyl arginine deiminase (PAD) which is the human equivalent of this enzyme implicated as a susceptibility factor for RA. It is also known that, individuals with periodontal infection due to Pg are exposed to the antigens generated by PAD, such as deaminated fibrin/fibrinogen, keratin and vimentin. The same antigens act as systemic immunogens in the production of rheumatoid factor as well as inflammation of the gingiva and synovial tissue locally [115, 116]. A clinical trial further reports that subjects with RA show some reduction in severity of RA, six weeks following non-surgical periodontal treatment [117]. Studies of cytokine profiles of subjects affected by RA and PD report that those with both diseases have raised titres of IL-10, IL- $1 \alpha$, TNF- $\alpha$, LT- $\alpha$ (lymphotoxin- $\alpha$ ), and low titres of auto-antibodies to IL-1 $\alpha$ and IL-6 [118]. Similarities in cytokine profiles in both RA and PD could also suggest a common underlying dysregulation of inflammatory and immune responses. Some genetic associations between RA and PD have also been suggested [119].

A high incidence of RA in subjects with PD has been documented, while some clinical trials report that subjects with RA experience moderate/severe PD, when compared with healthy individuals. It is relevant that a $P g$-induced extra-synovial inflammatory model contributed to the severity of experimental arthritis. The potential for genetic defects contributing to dyeregulated immunomodulation is common to both arthritis and periodontitis [120, 121]. This clinical correlation between RA and PD could be attributed to the common underlying pathobiology of both diseases.

Experimentally-induced inflammatory arthritis in rats has been linked to high levels of tissue MMPs, TNF- $\alpha$ and IL- $1 \beta$ in the synovial tissue as well as in gingival tissue. This study suggests that, RA and PD are closely related through a common dysregulation of inflammatory mechanisms in the two diseases [122].

Considering the robust biological mechanisms linking RA and PD, the therapeutic agents targeting these two diseases would be effective in reducing the levels of proinflammatory cytokines and destructive proteases implicated in the inflammatory pathogenic mechanisms of both diseases. 


\section{POTENTIAL ADJUNCTIVE ROLE OF TETRACY- CLINES IN RA AND PERIODONTITIS:}

Tetracycline with its desirable quality of anti-MMP activity is valuable in subjects with RA. Clinical trials report that minocycline administration in subjects with RA, was associated with significant reduction in disease activity [123]. At the same time, soft and hard tissue destruction in PD is partly due to bacterial virulent factors such as enzymes and partly due to MMPs. Tetracyclines and their analogs have effectively been used to combat more aggressive, rapidly progressive and refractory types of periodontitis. They act not only by suppressing the growth of pathogenic organisms responsible for PD, but also by inhibiting collagen degradation in connective tissues of the periodontal ligament and gingivae; as well as by decreasing the destruction of alveolar bone, by inhibiting MMPs [29].

An increasing understanding of the importance of MMPs in the pathogenesis of PD has led to the development of SDD at $20 \mathrm{mg} \mathrm{b.d}$, to modulate the inflammatory response by inhibiting MMP levels [71]. Based on many clinical trials carried out in the recent past, it has been reported that the two semi-synthetic antimicrobial tetracyclines (minocycline and doxycycline) have modest clinical benefits in RA [124]. CMTs [doxycycline (CMT-3) and minocycline (CMT-6)], not only inhibit the synthesis of MMPs, but are potent inhibitors of osteoclastogenesis in vitro $[125,126]$. CMT- 8 has also been shown to exert anti-inflammatory effects by inhibiting nitric oxide (NO) synthesis; and it can modify cell viability by exerting strong anti-apoptotic activity [127]. Thus, these CMTs may retard tissue break down and bone resorption in RA and PD, which could emerge as diseasemodifying drugs for both diseases. Semi-synthetic tetracyclines have been identified previously as inhibitors of angiogenesis, by preventing epithelial cell growth and inhibiting MMP synthesis.

The above reports clarify the fact that tetracyclines have significant therapeutic applications in inflammatory diseases associated with vascular tissue development and abnormal expression of MMPs. This phenomenon is relevant to explain the pathological conditions in soft tissue, cartilage and bone, which have close associations and similarities in PD and RA.

The results of two previous open trials and three doubleblind controlled studies indicate that early administration of minocycline could be beneficial to subjects with RA, or those with mild disease [128]; in the context of joint swelling, tenderness, laboratory parameters and patient assessments. These beneficial effects were confirmed in a metaanalysis summarizing clinical trials that had been conducted until then [123]. Despite these findings and the FDA approval of semi-synthetic tetracyclines as medications for RA, a recent review acknowledged that there are other agents that surpass the relatively weaker anti-inflammatory effects of tetracyclines [124]. However, tetracyclines have applications in osteoarthritis; prevention of cartilage degradation in vitro by minocycline is impressive, providing rationale for their applications.

Secretory phospholipase A2 (sPLA2) plays an important role during inflammation and has been implicated in articular inflammatory pathogenesis of RA. Minocycline has been shown to inhibit a calcium-dependent pathway of the enzyme by blocking the substrate interactive site. The dimethylamino group at $\mathrm{C} 7$ of minocycline could play a significant role in this interaction by linking with hydrophobic residues of the enzyme [129]. Tetracyclines also have the advantage of readily penetrating cell membrane barriers, unlike other highly charged inhibitors of PLA2. Over-expression of NOS is a characteristic feature of several chronic inflammatory autoimmune diseases. Its modulation by minocycline by making the iNOS mRNA susceptible to degradation provides a new therapeutic approach, seen in RA and experimental colitis. This could be non-specific [130]. Excessive MMP activity is linked to the pathophysiology of several diseases such as CHD, RA and inflammatory bowel disease [131].

Osteoclasts and dendritic cells share common progenitors in bone marrow-derived macrophages. Tetracyclines convert the differentiation pathway resulting in cells resembling dendritic cells and not osteoclasts [132]. Both doxycycline and minocycline inhibit osteoclastogenesis induced by receptor activator of NF-kB ligand (RANKL), with no effects on cell growth, phagocytic activity; or proliferation and differentiation of osteoblasts. RANKL-induced osteoclastogenesis was inhibited by in vivo injection of tetracyclines into RANKL-injected mice and RANKL-transgenic mice; at the same time, it promoted the appearance of $\mathrm{CD} 11 \mathrm{c}(+)$ cells. MAPK and c-Fos signals are essential for osteoclastic differentiation. There was inhibition of phosphorylation of MAPKs and c-Fos expression in bone marrow macrophages; this data suggests that the inhibition of MAPK and c-Fos signaling by tetracyclines is essential for preventing osteoclastic differentiation. These findings suggest a mechanism for prevention of bone loss by tetracyclines in response to local inflammation, as seen in periodontitis and RA; by conversion of osteoclastic differentiation resulting in a dendritic cell type. They have applications as potential agents for preventing bone resorption. The development of drug delivery systems would facilitate their use in preventing bone loss in response to inflammation induced by periodontitis and arthritis.

Osteoarthritis results in degeneration of articular cartilage; preclinical data suggest that doxycycline could slow down cartilage degeneration and act as a disease-modifying agent for the treatment of osteoarthritis. A Cochrane review examined the effects of doxycycline compared with placebo/no intervention on pain and function in subjects with osteoarthritis of the hip or knee [133], and confirmed the above findings. There has been progressive evidence in recent years to suggest an association between PD and increased risk of RA. Inflammatory mediators as well as microbial endotoxins have been suggested as plausible channels linking the two diseases. However, therapeutic measures utilized to inhibit common inflammatory mediators and effector molecules such as MMPs could reduce the severity of both diseases [134].

\section{NON-STEROIDAL ANTI-INFLAMMATORY DRUGS:}

Non-steroidal anti-inflammatory drugs (NSAIDs) have been widely used in the management of RA to control inflammation and pain. While the principal mechanism by 
which NSAIDs act is by inhibiting cycloxygenase responsible for prostaglandins (PG), it is also documented that NSAIDs can directly inhibit the activation and function of neutrophils; they also inhibit the release of TNF- $\alpha$ from monocytes and non-killer cells [135]. Consistent with previous studies on animal models of chronic destructive diseases such as RA, combination therapy with SDD and NSAID synergistically suppresses MMP and other neutral proteases in the gingivae of periodontitis subjects [136]. One of the mechanisms suggested is that the NSAID flurbiprofen in the combination regimen, enhances the uptake of SDD in the inflammatory lesion, thus synergistically reinforcing the effects of the medication. Inclusion of NSAIDs as adjuncts to the treatment protocols of PD could also be beneficial for arthritis presenting as a comorbidity, for their long-term management.

Cycloxygenase- independent effects are also believed to contribute towards the efficacy of NSAIDs in the treatment of RA. Many studies report that, periodontally-diseased tissues have higher PG levels, especially $\mathrm{PGE}_{2}$, than healthy tissues and are potent mediators of inflammation [137]. Bone resorption in PD is partly mediated by PGs which play an important role in its progression. As PGs are important mediators of bone resorption in PD, the use of NSAIDs would be beneficial in preventing inflammation-induced bone loss. $\mathrm{PGE}_{2}$ is a potent mediator of several chronic inflammatory diseases, including periodontitis. The inducible enzyme microsomal PGE synthase-1 (mPGES-1) catalyzing the terminal step of $\mathrm{PGE}_{2}$ biosynthesis is an attractive target for $\mathrm{PGE}_{2}$ inhibition. Aminothiazoles (heterocyclic amines) were effective in reducing inflammation and alveolar bone loss by $46 \%$ in an experimental periodontitis model [138]; they represent novel PGES-1 inhibitors which could have applications as anti-inflammatory agents in chronic inflammatory diseases with similar pathogeneses as periodontitis, by inhibiting $\mathrm{PGE}_{2}$.

Gingival and oral periosteal fibroblasts enhance yields of the antioxidant marker DHT (5 $\alpha$-dihydrotestosterone) relevant to wound healing in gingival and periosteal fibroblasts in response to minocycline, oestradiol, indomethacin and their combinations [139]. Adjunctive periodontal treatment with minocycline or indomethacin could contribute to wound healing in the inflamed periodontium. Reduced yields of DHT in response to the alkaline phosphatase (ALP) inhibitor levamisole were restored by indomethacin [140]. Enhanced ALP activity in response to indomethacin has implications for an adjunctive role in healing responses in periodontitis. Ketorolac tromethamine gel containing genipin was found to be effective against gingivitis in the periodontal pocket, by enhancing anti-inflammatory activity and increased crosslinking of genipin with biological tissue [141].

\section{CONJUGATED LINOLEIC ACID}

Conjugated linoleic acid (CLA) has also been found to be an important inhibitor of osteoclastogenesis. While CLA acts by modulating the RANK-L signaling pathway, it has been shown to positively influence calcium and bone metabolism [142]. This study suggests important therapeutic implications in the treatment of inflammatory diseases associated with bone destruction.

\section{BISPHOSPHONATES}

Since osteoclasts are responsible for the resorption and removal of bone, certain agents such as bisphosphonates which affect osteoclast function could be effective in the treatment of both PD and RA. Focal bone damage and generalized bone loss are features of RA, and studies have shown that, zoledronic acid which is a new generation bisphosphonate could reduce new bony erosions in patients with RA [143, 144]. The above findings suggest the beneficial effects of bisphosphonates in controlling these chronic inflammatory diseases in patients. Alveolar bone loss is an important complication of the inflammatory process in periodontitis where markers of inflammation stimulate osteoclastic bone resorption. In the context of inflammatory agents, lymphotoxin- $\alpha$ is considered to be the closest homolog to TNF- $\alpha$ [145]. Bisphosphonate therapy is considered to be beneficial as it inhibits bone resorption and increases bone mass. It also improves clinical parameters in subjects with periodontitis, and can be considered as a useful adjunct in the management of PD [146].

\section{ORNIDAZOLE}

Ornidazole which is a synthetic nitroimidazole with potent antiprotozoal and antibacterial activities has been identified as an effective agent against many periodontal pathogens, relevant to the treatment of PD [147]. Similar therapeutic benefits have been documented with ornidazole in the treatment of patients with RA. Administration of ornidazole in subjects with active RA has led to a significant reduction in arthritic pain, C-reactive protein (CRP) and ESR levels as well as reduction in other symptoms such as the duration of morning joint stiffness experienced by the patients. Common inflammatory pathways and similarities between the periodontium and joint synovium make interesting comparisons between these disease entities in the context of pathogeneses and treatment outcome, targeting inflammatory foci.

\section{SUMMARY AND DISCUSSION}

The MMP-inhibitory actions of tetracyclines and hostmodulatory effects, independent of their antimicrobial actions, were first reported in 1983. Subsequently, specific non-antimicrobial formulations of tetracyclines such as SDD $[3,33,40]$ and CMTs were developed as effective adjuncts in the management of chronic inflammatory disorders [6]. The rationale for the applications of SDD mainly for periodontitis and dermatological conditions; and CMTs particularly the most potent CMT-3, led to the discovery of the active site for MMP inhibition in the molecule. The diverse applications for CMT-3 [4] include acute respiratory distress syndrome, Kaposi's sarcoma and its anti-angiogenesis effects in cancer therapy. In addition, the safety and effective therapeutic outcome in periodontitis, other oral diseases; and systemic conditions encompassing RA, post-menopausal osteopenia, type 2 DM, CHD and others are addressed [4549] and [67]. Their applications in this context, in view of inflammatory risk markers in common with periodontitis, have been discussed throughout relevant sections. 
Beneficial and safety aspects of minocycline alone or in combination with other medications in diseases with an inflammatory pathogenesis have been confirmed in diverse animal models and some clinical trials [10]. Its multiple mechanisms of action affect several targets, for antioxidant effects, chelation of calcium; and inhibition of proinflammatory enzymes such as iNOS and MMPs; which play an important role in attenuating the inflammatory diseases addressed. Although doxycycline has been reported to share some of these properties [22], a similar efficacy has not been demonstrated in the same models. Its multiple targets make minocycline significantly effective amongst tetracyclines $[23,26]$. It is also safe, well absorbed orally, welldistributed, relatively inexpensive for long-term use and its side-effects are well-documented. Further work would clarify its mechanisms of action in vivo. However, the scope and diversity of minocycline make it a useful therapeutic adjunct in the management of several diseases including periodontitis and prevalent comorbidities addressed here [50, 51]. Minocycline has long been used as an antibiotic; there is increasing documentation of its anti-inflammatory, antiapoptotic, immunomodulatory and neuroprotective actions which have attracted a great deal of interest in view of its therapeutic potential, independent of its antimicrobial effects.

Minocycline crosses cell membranes readily and has potent anti-apoptotic actions; doxycycline is an effective antiprotease. They demonstrate radical scavenging activity and overcome oxidative stress. They belong to a phenolic class of antioxidants and have properties consistent with their structure, being similar to vitamin E. Radical scavenging actions of minocycline are similar to those of tocopherol [13, 14]. It is effective as a superoxide scavenger in quenching hydrogen peroxide and peroxynitrite by direct action on free radicals. The diethyl amino group on the phenolic carbon of the minocycline molecule provides improved steric hindrance and confers superior radical scavenging activity on minocycline; being 200-300 fold as potent as tetracycline. It is also equally potent as tetracycline in its inhibition of lipid peroxidation.

These positive actions extend beyond stabilization of periodontitis. Decreased inflammatory loading could contribute to decreased osteopenia and inflammatory risk markers in CHD, DM and arthritis, considering the prevalence of common markers for disease progression across these entities and periodontitis [46, 83, 84, 92, 109, 122, 123] and [128]. SDD is a more potent inhibitor of MMPs than tetracycline; and safer than minocycline with superior pharmacokinetics which favor patient compliance. Their actions in stimulating osteoblasts and enhancing the formation of mineralized bone matrix in response to long-term exposure complement their therapeutic efficacy in the adjunctive management of periodontitis [22-25, 33, 34, 41]. As discussed throughout this paper, these actions could have positive effects on prevalent comorbidities in periodontitis subjects, when used as therapeutic adjuncts.

\section{ABBREVIATIONS}

ACS $=$ acute coronary syndrome

ACVD $=$ atherosclerotic cardiovascular disease

$\begin{array}{ll}\text { AGE } & =\text { advanced glycemic end products } \\ \text { CAL } & =\text { clinical attachment level } \\ \text { CHD } & =\text { chemically modified tetracycline. } \\ \text { CMT } & =\text { C-reactive protein } \\ \text { CRP } & =\text { diabetes mellitus } \\ \text { DM } & =\text { gingival crevicular fluid } \\ \text { GCF } & =\text { high density lipoprotein } \\ \text { HLD } & =\text { interleukin } \\ \text { IL } & =\text { low density lipoprotein } \\ \text { LDL } & =\text { lipopolysaccharide } \\ \text { LPS } & =\text { macrophage chemoattractant protein-1 } \\ \text { MCP-1 } & =\text { metabolic syndrome } \\ \text { MetS } & =\text { myocardial infarct } \\ \text { MI } & \text { V }\end{array}$

\section{CONFLICT OF INTEREST}

There is no potential conflict of interest associated with the contents.

\section{ACKNOWLEDGEMENTS}

Both authors contributed to literature searches and writing of the paper.

\section{REFERENCES}

[1] Golub LM. Introduction and background. Pharmacol Res 2011; 63: 99-101. 
[2] Choi DH, Moon IS, Choi BK, et al. Effects of sub-antimicrobial dose of doxycycline therapy on crevicular fluid MMP-8 and gingival tissue MMP-9, TIMP-1 and IL-6 levels in chronic periodontitis. J Periodontal Res 2004; 39: 20-6.

[3] Payne JB, Golub LM. Using tetracyclines to treat osteoporotic/osteopenic bone loss: from the basic science laboratory to the clinic. Pharmacol Res 2011; 63: 121-9.

[4] Marcial BL, Sousa SF, Barbosa IL, Dos Santos HF, Ramos MJ. Chemically modified tetracyclines as inhibitors of MMP-2 matrix metalloproteinase: a molecular and structural study. J Phys Chem B 2012; 116: 13644-54.

[5] Roy SK, Kubiak BD, Albert SP, et al. Chemically-modified tetracycline-3 prevents acute respiratory distress syndrome in a porcine model of sepsis plus ischemia/reperfusion lung injury. Shock 2012; 37: 424-32.

[6] Garrido-Mesa N, Zarzuelo A, Gálvez J. Minocycline: far beyond an antibiotic. Br J Pharmacol 2013; 169: 337-52.

[7] Johnston S. Feeling blue? Minocycline-induced staining of the teeth, oral mucosa, sclerae and ears: a case report. Br Dent J 2013; 215: 71-3.

[8] Zhang Y, Gu Y, Lee HM, et al. Design, synthesis and biological activity of new polyenolic inhibitors of matrix metalloproteinases: a focus on chemically-modified curcumins. Curr Med Chem 2012; 19: 4348-58.

[9] Devy L, Dransfield DT. New strategies for the next generation of matrix-metalloproteinase inhibitors: selectively targeting membrane-anchored MMPs with therapeutic antibodies. Biochem Res Int 2011; 2011: 191670.

[10] Garrido-Mesa N, Zarzuelo A, Gálvez J. What is behind the nonantibiotic properties of minocycline? Pharmacol Res 2013; 67: 1830 .

[11] Griffin MO, Ceballos G, Villarreal FJ. Tetracycline compounds with non-antimicrobial organ protective properties: possible mechanisms of action. Pharmacol Res 2011; 63: 102-7.

[12] Webster G, Del Rosso JQ. Anti-inflammatory activity of tetracyclines. Dermatol Clin 2007; 25: 133-5.

[13] Kraus RL, Pasieczny R, Lariosa-Willingham K, Turner MS, Jiang a, Trauger JW. Antioxidant properties of minocycline: neuroprotection in an oxidative stress assay and direct radical-scavenging activity. J Neurochem 2005; 94: 819-27.

[14] Leite LM, Carvalho AGG, Tavares Ferreira PLF, et al. Antiinflammatory properties of doxycycline and minocycline in experimental models: an in vivo and in vitro comparative study. Inflammopharmacology 2011; 19: 99-110.

[15] Yenari MA, Xu L, Tang XN, Qiao Y, Giffard RG. Microglia potentiate damage to blood-brain barrier constituents: improvement by minocycline in vivo and in vitro. Stroke 2006; 37: 1087-93.

[16] Sonmez E, Kabatas S, Ozen O, et al. Minocycline treatment inhibits lipid peroxidation, preserves spinal cord ultrastructure and improves functional outcome after traumatic spinal cord injury in the rat. Spine 2013; 38: 1253-9.

[17] Takashiba S, Naruishi K, Murayama Y. Perspective of cytokine regulation for periodontal treatment: fibroblast biology. J Periodontol 2003; 74: 103-10.

[18] El-Shinnawi U, Soory M. Associations between periodontitis and systemic inflammatory diseases: response to treatment. Recent Pat Endocr Metab Immune Drug Discov 2013; 7: 169-88.

[19] Caton J, Ryan ME. Clinical studies on the management of periodontal diseases utilizing subantimicrobial dose doxycycline (SDD). Pharmacol Res 2011; 63: 114-20.

[20] Serhan CN. Controlling the resolution of acute inflammation: a new genus of dual anti-inflammatory and proresolving mediators. $J$ Periodontol 2008; 79: 1520-6.

[21] Gu Y, Walker C, Ryan ME, Payne JB, Golub LM. Nonantibacterial tetracycline formulations: clinical applications in dentistry and medicine. J Oral Microbiol 2012; 4 . doi: 10.3402/jom.v4i0.19227. Epub 2012 Oct 12.

[22] Moreno Villagrana AP, Gomez Clavel JF. Antimicrobial or sub antimicrobial antibiotic therapy as an adjunct to the nonsurgical periodontal treatment: a meta-analysis. ISRN Dent 2012; 2012: 581207, 11 pp. doi: 10.5402/2012/581207. Epub 2012 Oct 22.

[23] Gomes PS, Fernandes MH. Effect of therapeutic levels of doxycycline and minocycline in the proliferation and differentiation of human bone marrow osteoblastic cells. Arch Oral Biol 2007; 52: 251-9.
[24] Kirkwood KL, Cirelli JA, Rogers JE, Giannobile WV. Novel host response therapeutic approaches to treat periodontal diseases. Periodontol 2000, 2007; 43: 294-315.

[25] Basegmez C, Berber L, Yalcin F. Clinical and biochemical efficacy of minocycline in non-surgical periodontal therapy: a randomized controlled pilot study. J Clin Pharmacol 2011; 51: 915-22.

[26] Honibald EN, Mathew S, Padmanaban J, Sundaram E, Ramamoorthy RD. Perioceutics: Matrix metalloproteinase inhibitors as an adjunctive therapy for inflammatory periodontal disease. J Pharm Bioallied Sci 2012; 4 (Suppl 2): S417-21.

[27] Giannobile WV. Host-response therapeutics for periodontal diseases. J Periodontol 2008; 79: 1592-600.

[28] Ashley RA. Clinical trials of a matrix metalloproteinase inhibitor in human periodontal disease. SDD Clinical Research Team. Ann NY Acad Sci 1999; 878: 335-46.

[29] Sorsa T, Tjäderhane L, Konttinen YT, et al. Matrix metalloproteinases: contribution to pathogenesis, diagnosis and treatment of periodontal inflammation. Ann Med 2006; 38: 306-21.

[30] Ciancio S, Ashley R. Safety and efficacy of subantimicrobial-dose doxycycline therapy in patients with adult periodontitis. Adv Dent Res 1998; 12: 27-31.

[31] Caton JG, Ciancio SG, Blieden TM, et al. Treatment with subantimicrobial dose doxycycline improves the efficacy of scaling and root planing in patients with adult periodontitis. J Periodontol 2000; 71: 521-32.

[32] Agnihotri R, Gaur S. Chemically modified tetracyclines: novel therapeutic agents in the management of chronic periodontitis. Indian J Pharmacol 2012; 44: 161-7.

[33] Reddy MS, Geurs NC, Gunsolley JC. Periodontal host modulation with antiproteinase, anti-inflammatory, and bone-sparing agents: a systematic review. Ann Periodontol 2003; 8: 12-37.

[34] Preshaw PM, Novak MJ, Mellonig J, et al. Modified release subantimicrobial dose doxycycline enhances scaling and root planing in subjects with periodontal disease. J Periodontol 2008; 79: 440-52.

[35] Gapski R, Barr JL, Sarment DP, Layher MG, Socransky SS, Giannobile WV. Effect of systemic matrix metalloproteinase inhibition on periodontal wound repair: a proof of concept trial. J Periodontol 2004; 75: 441-52.

[36] Golub LM, Lee HM, Stoner JA, et al. Doxycycline effects on serum bone biomarkers in post-menopausal women. J Dent Res 2010; 89: 644-9.

[37] Reinhardt RA, Stoner JA, Golub LM, et al. Efficacy of subantimicrobial dose doxycycline in post-menopausal women: clinical outcomes. J Clin Periodontol 2007; 34: 768-75.

[38] Golub LM, Lee HM, Stoner JA, et al. Subantimicrobial dose doxycycline modulates gingival crevicular fluid biomarkers of periodontitis in postmenopausal osteopenic women. J Periodontol 2008; 79: 1409-18.

[39] Payne JB, Golub LM, Stoner JA, et al. The effect of subantimicrobial-dose-doxycycline periodontal therapy on serum biomarkers of systemic inflammation. J Am Dent Assoc 2011; 142: 262-73.

[40] Kim SE, Kim S, Jeong M, et al. Experimental determination of a sub antimicrobial dosage of doxycycline hyclate for treatment of periodontitis in Beagles. Am J Vet Res 2013; 74: 130-5.

[41] Sgolastra F, Petrucci A, Gatto R, Giannoni M, Monaco A. Longterm efficacy of subantimicrobial-dose doxycycline as an adjunctive treatment to scaling and root planing: a systematic review and meta-analysis. J Periodontol 2011; 82: 1570-81.

[42] Monk E, Shalita A, Siegel DM. Clinical applications of nonantimicrobial tetracyclines in dermatology. Pharmacol Res 2011; 63: $130-45$.

[43] Sapadin AN, Fleischmajer R. Tetracyclines: nonantibiotic properties and their clinical implications. J Am Acad Dermatol 2006; 54 : 258-65.

[44] Ryan ME, Ramamurthy NS, Sorsa T, Golub LM. MMP mediated events in diabetes. Ann NY Acad Sci 1999; 878: 311-34.

[45] Garrido-Mesa N, Camuesco D, Arribas B, et al. The intestinal anti-inflammatory effect of minocycline in experimental colitis involves both its immunomodulatory and antimicrobial properties. Pharmacol Res 2011; 63: 308-19.

[46] Cai Z, Zhao Y, Yao S, Bin Zhao B. Increases in $\beta$-amyloid protein in the hippocampus caused by diabetic metabolic disorder are blocked by minocycline through inhibition of NF- $\kappa$ B pathway activation. Pharmacol Rep 2011; 63: 381-91.

[47] Scarabelli TM, Stephanou A, Pasini E, et al. Minocycline inhibits caspase activation and reactivation, increases the ratio of XIAP to 
smac/DIABLO, and reduces the mitochondrial leakage of cytochrome $\mathrm{C}$ and smac/DIABLO. J Am Coll Cardiol 2004; 43: 86574.

[48] Bench TJ, Jeremias A, Brown DL. Matrix metalloproteinase inhibition with tetracyclines for the treatment of coronary artery disease. Pharmacol Res 2011; 64: 561-6.

[49] Alano CC, Kauppinen TM, Valls AV, Swanson RA. Minocycline inhibits poly(ADP-ribose) polymerase-1 at nanomolar concentrations. Proc Natl Acad Sci USA 2006; 103: 9685-90.

[50] Nikodemova M, Duncan ID, Watters JJ. Minocycline exerts inhibitory effects on multiple mitogen-activated protein kinases and IкB $\alpha$ degradation in a stimulus-specific manner in microglia. J Neurochem 2006; 96: 314-23.

[51] Henry CJ, Huang Y, Wynne A, et al. Minocycline attenuates lipopolysaccharide (LPS)-induced neuroinflammation, sickness behavior, and anhedonia. J Neuroinflamm 2008; 5: 15.

[52] Park CH, Shin TK, Lee HY, Kim SJ, Lee WS. Matrix metalloproteinase inhibitors attenuate neuroinflammation following focal cerebral ischemia in mice. Korean J Physiol Pharmacol 2011; 15: 11522.

[53] Markovic DS, Vinnakota K, van Rooijen N, et al. Minocycline reduces glioma expansion and invasion by attenuating microglial MT1-MMP expression. Brain Behav Immun 2011; 25: 624-8.

[54] Liu WT, Lin CH, Hsiao M, Gean PW. Minocycline inhibits the growth of glioma by inducing autophagy. Autophagy 2011; 7: 16675.

[55] Huang TY, Chu HC, Lin YL, et al. Minocycline attenuates experimental colitis in mice by blocking expression of inducible nitric oxide synthase and matrix metalloproteinases. Toxicol Appl Pharmacol 2009; 237: 69-82.

[56] Perencevich M, Burakoff R. Use of antibiotics in the treatment of inflammatory bowel disease. Inflamm Bowel Dis 2006; 12: 651-64.

[57] Schultz M. Clinical use of E. coli Nissle 1917 in inflammatory bowel disease. Inflamm Bowel Dis 2008; 14: 1012-8.

[58] Garrido-Mesa N, Utrilla P, Comalada M, et al. The association of minocycline and the probiotic Escherichia coli Nissle 1917 results in an additive beneficial effect in a DSS model of reactivated colitis in mice. Biochem Pharmacol 2011; 82: 1891-900.

[59] Yong VW, Wells J, Giuliani F, Casha S, Power C, Metz LM. The promise of minocycline in neurology. Lancet Neurol 2004; 3: 74451.

[60] Stirling DP, Koochesfahani KM, Steeves JD, Tetzlaff W. Minocycline as a neuroprotective agent. Neuroscientist 2005; 11: 30822 .

[61] Sutton TA, Kelly KJ, Mang HE, Plotkin Z, Sandoval RM, Dagher PC. Minocycline reduces renal microvascular leakage in a rat model of ischemic renal injury. Am J Physiol Renal Physiol 2005; 288: F91-F7.

[62] Jordan J, Fernandez-Gomez FJ, Ramos M, Ikuta I, Aguirre N, Galindo MF. Minocycline and cytoprotection: shedding new light on a shadowy controversy. Curr Drug Deliv 2007; 4: 225-31.

[63] Lampl Y, Boaz M, Gilad R, et al. Minocycline treatment in acute stroke: an open-label, evaluator-blinded study. Neurol 2007; 69: 1404-10.

[64] Andrassy M, Volz HC, Igwe JC, et al. High-mobility group box-1 in ischemia-reperfusion injury of the heart. Circulation 2008; 117: 3216-26.

[65] Hu X, Zhou X, He B, et al. Minocycline protects against myocardial ischemia and reperfusion injury by inhibiting high mobility group box 1 protein in rats. Eur J Pharmacol 2010; 638: 84-9.

[66] Tao R, Kim SH, Honbo N, Karliner JS, Alano CC. Minocycline protects cardiac myocytes against simulated ischemia-reperfusion injury by inhibiting poly (ADP-ribose) polymerase-1. J Cardiovasc Pharmacol 2010; 56: 659-68.

[67] Shahzad K, Thati M, Wang H, et al. Minocycline reduces plaque size in diet induced atherosclerosis via p27(Kip1). Atherosclerosis 2011; 219: 74-83.

[68] Gu Y, Lee HM, Sorsa T, et al. Non-antibacterial tetracyclines modulate mediators of periodontitis and atherosclerotic cardiovascular disease: a mechanistic link between local and systemic inflammation. Pharmacol Res 2011; 64: 573-9.

[69] Alfakry H, Sinisalo J, Paju S, et al. The association of serum neutrophil markers and acute coronary syndrome. Scand J Immunol 2012; 76: 181-7.

[70] Sorsa T, Tervahartiala T, Leppilahti J, et al. Collagenase-2 (MMP8 ) as a point-of-care biomarker in periodontitis and cardiovascular diseases. Therapeutic response to non-antimicrobial properties of tetracyclines. Pharmacol Res 2011; 63: 108-13.

[71] Preshaw PM: Warnakulasuriya S and Tilakaratne WM, Ed. In: Oral Medicine \& Pathology: a guide to diagnosis and management; Chapter 3: Periodontal disease. 1st ed. Jaypee Brothers Medical Publishers New Delhi 2014; 50-81. ISBN: 978-93502522215.

[72] Marchetti E, Monaco A, Procaccini L, et al. Periodontal disease: the influence of metabolic syndrome. Nutr Metab 2012; 9: 88 doi: 10.1186/1743-7075-9-88.

[73] Merchant AT, Pitiphat W, Rimm EB, Joshipura K. Increased physical activity decreases periodontitis risk in men. Eur J Epidemiol 2003; 18: 891-8.

[74] Alikhani M, Alikhani Z, Boyd C, et al. Advanced glycation end products stimulate osteoblast apoptosis via MAP kinase and cytosolic apoptotic pathways. Bone 2007; 40: 345-53.

[75] Borges I Jr, Moreira EA, Filho DW, de Oliveira TB, da Silva MB, Frode TS. Pro-inflammatory and oxidative stress markers in patients with periodontal disease. Mediators Inflamm 2007; 4: 57-94.

[76] Montebugnoli L, Servidio D, Miaton RA, Prati C, Tricoci P, Melloni C. Poor oral health is associated with coronary heart disease and elevated systemic inflammatory and haemostatic factors. J Clin Periodontol 2004; 31: 25-9.

[77] Al-Zahrani MS, Borawski EA, Bissada NF. Periodontitis and three health-enhancing behaviours: maintaining normal weight, engaging in recommended level of exercise and consuming a high quality diet. J Periodontol 2005; 76: 1362-6.

[78] Pischon T, Hankinson SE, Hotamisligil GS, Rifai N, Rim EB Leisure time physical activity and reduced plasma levels of obesity-related inflammatory markers. Obes Res 2003; 11: 1055-64.

[79] Hansel B, Giral P, Nobecourt E, et al. Metabolic syndrome is associated with elevated oxidative stress and dysfunctional dense highdensity lipoprotein particles displaying impaired antioxidative activity. J Clin Endocrinol Metab 2004; 89: 4963-71.

[80] Koyama H, Shoji T, Yokoyama M, et al. Plasma level of endogenous secretory RAGE is associated with components of the metabolic syndrome and atherosclerosis. Arterioscler Thromb Vasc Biol 2005; 25: 2587-93.

[81] Basta G, Schmidt AM, Decaterina R. Advanced glycation end products and vascular inflammation: implications for accelerated atherosclerosis in diabetes. Cardiovasc Res 2004; 63: 582-92.

[82] Castro MM, Kandasamy AD, Youssef N, Schulz R. Matrix metalloproteinase inhibitor properties of tetracyclines: therapeutic potential in cardiovascular disease. Pharmacol Res 2011; 64: 551-60.

[83] Brown DL, Desai KK, Vakili BA, Nouneh C, Lee HM, Golub LM. Clinical and biochemical results of the metalloproteinase inhibition with subantimicrobial doses of doxycycline to prevent acute coronary syndromes (MIDAS) pilot trial. Arterioscler Thromb Vasc Biol 2004; 24: 733-8.

[84] Ridker PM, Danielson E, Fonseca FA, et al. Rosuvastatin to prevent vascular events in men and women with elevated $\mathrm{C}$ reactive protein. N Engl J Med 2008; 359: 2195-207.

[85] Salminen A, Pussinen PJ, Payne JB, et al. Subantimicrobial-dose doxycycline treatment increases serum cholesterol efflux capacity from macrophages. Inflamm Res 2013; 62: 711-20.

[86] Axisa B, Loftus IM, Naylor AR, et al. Prospective, randomized, double-blind trial investigating the effect of doxycycline on matrix metalloproteinase expression within atherosclerotic carotid plaques. Stroke 2002; 33: 2858-64

[87] Bendeck MP, Conte M, Zhang M, Nili N, Strauss BH, Farwell SM. Doxycycline modulates smooth muscle cell growth, migration, and matrix remodelling after arterial lesions. Am J Pathol 2002; 160: 1089-95

[88] Baxter BT, Pearce WH, Waltke EA, et al. Prolonged administration of doxycycline in patients with small asymptomatic aortic aneurysms: report of a prospective (Phase II) multi-centre study. J Vasc Surg 2002; 36: 1-12.

[89] Ludvig DS. The glycemic index physiological mechanisms relating to obesity, diabetes and cardiovascular disease. JAMA 2002; 287: 2414-23.

[90] Janket SJ, Jones JA, Meurman JH, Baird AE, Van Dyke TE. Oral Infection, hyperglycemia and endothelial dysfunction. Oral Sur Oral Med Oral Pathol Oral Radiol Endocrinol 2008; 105: 173-9.

[91] Nakao C, Angel M, Di Mateo S, Komesu MC. Effects of topical tetracycline in wound healing on experimental diabetes in rats. Open Diabetes J 2009; 2: 53-9. 
[92] Mustapha IZ, Debrey S, Oladubu M, Ugarte R. Markers of systemic bacterial exposure in periodontal disease and cardiovascular disease risk: a systematic review and meta-analysis. J Periodontol 2007; 78: 2289-02.

[93] Grundy SM. Metabolic syndrome scientific statement by the American Heart Association and the National Heart, Lung and Blood Institute. Atheroscler Thromb Vasc Biol 2005; 25: 2243-4.

[94] Fukui N, Shimazaki Y, Shinagawa T, Yamashita Y. Periodontal status and metabolic syndrome in middle-aged Japanese. J Periodontol 2012; 83: 1363-71.

[95] Kwon YE, Ha JE, Paik DI, Jin BH, Bae KH. The relationship between periodontitis and metabolic syndrome among a Korean nationally representative sample of adults. J Clin Periodontol 2011; 38: 781-6.

[96] Kushiyama M, Shimazaki Y, Yamashita Y. Relationship between metabolic syndrome and periodontal disease in Japanese adults. J Periodontol 2009; 80: 1610-5.

[97] D'Aiuto F, Sabbah W, Netuveli G, et al. Association of nthe metabolic syndrome with severe periodontitis in a large US populationbased survey. J Clin Endocrinol Metab 2008; 93: 3989-94.

[98] Suvan J, D'Aiuto F, Moles DR, Petrie A, Donos N. Association between overweight/obesity and periodontitis in adults: a systematic review. Obesity Reviews 2011; 12: e404-e81.

[99] Chaffee BW, Weston SJ. Association between chronic periodontal disease and obesity: a systematic review and meta-analysis. J Periodontol 2010; 81: 1708-24.

[100] Gorman A, Kaye EK, Apovian C, Fung TT, Nunn M, Garcia RI. Overweight and obesity predict time to periodontal periodontal disease progression in men. J Clin Periodontol 2012; 39: 107-14.

[101] Hirsh IB, Brownlee M. Should minimum blood glucose variability become the gold standard of glycemic control? J Diabetes Complicat 2005; 19: 178-81.

[102] Preshaw PM, Alba AL, Herrera D, et al. Periodontisis and diabetes: a two-way relationship. Diabetologia 2012; 55: 21-31.

[103] Esposito K, Nappo F, Marfella R, et al. Inflammatory cytokine concentrations are acutely increased by hyperglycemia in humans: role of oxidative stress. Circulation 2002; 106: 2067-72.

[104] Rahman ZA, Soory M. Antioxidant effects of glutathione and IGF in a hyperglycaemic cell culture model of fibroblasts: some actions of advanced glycaemic end products (AGE) and nicotine. Endocr Metab Immune Disord Drug Targets 2006; 6: 279-86.

[105] Tilakaratne A, Soory M. Osteoblastic responses to LPS, glucose oxidized LDL and minocycline: therapeutic targets for periodontal and cardiometabolic diseases. Recent Pat Endocr Metab Immune Drug Discov 2012; 6: 73-84.

[106] Grossi SG, Genco RJ. Periodontal disease and diabetes mellitus: a two-way relationship. Ann Periodontol 1998; 3: 51-61.

[107] Soory M, El-Shinnawi U: F. Veas, Ed. In: Acute phase proteins as early non-specific biomarkers of human and veterinary diseases. Chapter 3: Diagnostic value of acute phase proteins in periodontal, psychosomatic and cardiometabolic diseases: response to treatment. In Tech. $1^{\text {st }}$ ed, 2011; 43-68. ISBN 978-953-307-873-1.

[108] Engebretson SP, Hey-Hadavi J. Sub-antimicrobial doxycycline for periodontitis reduces hemoglobin A1c in subjects with type 2 diabetes: a pilot study. Pharmacol Res 2011; 64: 624-9.

[109] Emingil G, Gurkan A, Atilla G, Kantarci A. Sub-antimicrobialdose doxycycline and cytokine-chemokine levels in gingival crevicular fluid. J Periodontol 2011; 82: 452-61.

[110] Javed F, Al-Askar M, Al-Hezaimi K. Cytokine profile in the gingival crevicular fluid of periodontitis patients with and without type 2 diabetes: a literature review. J Periodontol 2012; 83: 156-61.

[111] Fell RA, Zeek Y, Arora M. The correlation of serum and gingival crevicular fluid cytokines in obese subjects. J Int Acad Periodontol 2013; 15, 20-8.

[112] Federici TJ. The non-antibiotic properties of tetracyclines: clinical potential in ophthalmic disease. Pharmacol Res 2011; 64: 614-23.

[113] Ogrendik M, Kokino S, Ozdemir F, Bird PS, Hamlet S. Serum antibodies to oral anaerobic bacteria in patients with rheumatoid arthritis. Med Gen Med 2005; 7: 2-10.

[114] Lundberg K, Kinloch A, Fisher BA, et al. Antibodies to citrullinated alpha-enolase peptide 1 are specific for rheumatoid arthritis and cross-react with bacterial enolase. Arthritis Rheum 2008; 58: 3009-19.

[115] Wegner N, Wait R, Sroka A, et al. Peptidylarginine deiminase from porphyromonas gingivalis citrullinates human fibrinogen and al- pha-enolase: Implications for autoimmunity in rheumatoid arthritis. Arthritis Rheum 2010; 62: 2662-72.

[116] Ortiz P, Bissada NF, Palomo L, et al. Periodontal therapy reduces the severity of active rheumatoid arthritis in patients treated with or without tumour necrosis factor inhibitors. J Periodontol 2009; 80: $535-40$.

[117] Havemose-Poulsen A, Serensen LK, Stoltze K, Bendtzen K, Holmstrup P. Cytokine profiles in peripheral blood and whole blood cell cultures associated with aggressive periodontitis, juvenile idiopathic arthritis and rheumatoid arthritis. J Periodontol 2005; 76: 2276-85.

[118] Marrotte H, Farge P, Gaudin P, Alexandre C, Mougin B, Moussec $\mathrm{P}$. The association between periodontal disease and joint destruction in rheumatoid arthritis extends the link between the HLA-DR shared epitopes. Ann Rheum Dis 2006; 65: 905-9.

[119] Bartold PM, Marino V, Cantley M, Haynes DR. Effect of Porphyromonas gingivalis-induced inflammation on the development of rheumatoid arthritis. J Clin Periodontol 2010; 37: 405-11.

[120] Ospelt C, Reedquist KA, Gay S, Tak PP. Inflammatory memories: is epigenetics the missing link to persistent stromal cell activation in rheumatoid arthritis? Autoimmun Rev 2011; 10: 519-24.

[121] Persson GR. Rheumatoid arthritis and periodontitis - inflammatory and infectious connections: review of the literature. J Oral Microbiol 2012; 4: 11829.

[122] Stone M, Fortin PR, Pacheco-Tena C, Inman RD. Should tetracycline treatment be used more extensively for rheumatoid arthritis? Meta-analysis demonstrates clinical benefit with reduction in disease activity. J Rheumatol 2003; 30: 2112-22.

[123] Greenwald RA. The road forward: the scientific basis for tetracycline treatment of arthritic disorders. Pharmacol Res 2011; 64: 6103.

[124] Suzuki A, Yagisawa J, Kumakura S, Tsutsui T. Effects of minocycline and doxycycline on cell survival and gene expression in human gingival and periodontal ligament cells. J Periodont Res 2006; 41: 124-31.

[125] Holmes SG, Still K, Buttle DJ, Bishop NJ, Grabowski PS. Chemically modified tetracyclines act through multiple mechanisms directly on osteoclast precursors. Bone 2004; 35: 471-8.

[126] D'Agostino P, Ferlazzo V, Milano S, et al. Anti-inflammatory effects of chemically modified tetracyclines by the inhibition of nitric oxide and interleukin-12 synthesis in J774 cell line. Int Immunopharmacol 2001; 1: 1765-76.

[127] Langevitz P, Livneh A, Bank I, Pras M. Benefits and risks of minocycline in rheumatoid arthritis. Drug Saf 2000; 22: 405-14.

[128] Dalm D, Palm GJ, Aleksandrov A, Simonson T, Hinrichs W. Nonantibiotic properties of tetracyclines: structural basis for inhibition of secretory phospholipase A2. J Mol Biol 2010; 398: 83-96.

[129] Dunston CR, Griffiths HR, Lambert PA, Staddon S, Vernallis AB. Proteomic analysis of the anti-inflammatory action of minocycline. Proteomics 2011; 11: 42-51.

[130] Gialeli C, Theocharis AD, Karamanos NK. Roles of matrix metalloproteinases in cancer progression and their pharmacological targeting. FEBS J 2011; 278: 16-27.

[131] Kinugawa S, Koide M, Kobayashi Y, et al. Tetracyclines convert the osteoclastic-differentiation pathway of progenitor cells to produce dendritic cell-like cells. J Immunol 2012; 188: 1772-81.

[132] da Costa BR, Nüesch E, Reichenbach S, Jüni P, Rutjes AW. Doxycycline for osteoarthritis of the knee or hip. Cochrane Database Syst Rev 2012 Nov 14; (11): CD007323. doi: 10.1002/14651858.CD007323.pub3.

[133] Modi DK, Chopra VS, Bhau U. Rheumatoid arthritis and periodontitis: Biological links and the emergence of dual purpose therapies. Indian J Dent Res 2009; 20: 86-90.

[134] Gunella G, Bardelli C, Spina S, Fresu LG, Viano I, Brunelleschi S. Anti-inflammatory drugs and tumour necrosis factor alpha production from monocytes: role of transcription factor NF- Kappa B and implications for rheumatoid arthritis therapy. Eur J Pharmacol 2006; 6: 199-208.

[135] Lee HM, Ciancio SG, Tüter G, Ryan ME, Komaroff E, Golub LM. Sub-antimicrobial dose doxycycline efficacy as a matrix metalloproteinase inhibitor in chronic periodontitis patients is enhanced when combined with a non-steroidal anti-inflammatory drug. J Periodontol 2004; 75: 453-63.

[136] Noguchi K, Ishikawa I. The roles of cyclooxygenase-2 and prostaglandin E2 in periodontal disease. Periodontol 2000 2007; 43: 85101. 
[137] Kats A, Båge T, Georgsson P, et al. Inhibition of microsomal prostaglandin E synthase-1 by aminothiazoles decreases prostaglandin E2 synthesis in vitro and ameliorates experimental periodontitis in vivo. FASEB J 2013; 27: 2328-41.

[138] Tilakaratne A, Soory M. The modulation of androgen metabolism by oestradiol, minocycline and indomethacin in a cell culture model. J Periodontol 2002; 73: 585-90.

[139] Soory M, Tilakaratne A. Androgen metabolic response to indomethacin and the alkaline phosphatase inhibitor levamisole in fibroblasts. J Clin Periodontol 2003; 30: 1069-74.

[140] Yang JH, Yun MY, Kim DK, et al. Preparation and evaluation of ketorolac tromethamine gel containing genipin for periodontal diseases. Arch Pharm Res 2007; 30: 871-5.

[141] Rahman MM, Bhattacharya A, Fernandes G. Conjugated linoleic acid inhibits osteoclast differentiation of RAW264.7 cells by modulating RANKL signalling. J Lipid Res 2006; 47: 1739-48.

[142] Breuil V, Euller-Ziegler L. Bisphosphonate therapy in rheumatoid arthritis. Joint Bone Spine 2006; 73: 349-54.
[143] Jarrett SJ, Conaghan PG, Sloan VS, et al. Preliminary evidence for a structural benefit of the new bisphosphonate zoledronic acid in early rheumatoid arthritis. Arthritis Rheum 2006; 54: 1410-4.

[144] Calmon-Hamaty F, Combe B, Hahne M, Morel J. Lymphotoxin $\alpha$ revisited: general features and implications in rheumatoid arthritis. Arthritis Res Ther 2011; 13: 232.

[145] Lane N, Armitage GC, Loomer P, et al. Bisphosphonate therapy improves the outcome of conventional periodontal treatment: results of a 12-month randomized placebo controlled study. J Periodontol 2005; 76: 1113-22.

[146] Kamma, JJ, Nakou M, Mitsis FJ. The clinical and microbiological effects of systemic ornidazole in sites with and without subgingival debridement in early-onset periodontitis patients. J Periodontol 2000; 71: 1862-73.

[147] Ogrendik M, Hakguder A, Keser N. Treatment of rheumatoid arthritis with ornidazole: a randomized double-blind placebocontrolled study. Rheumatology (Oxford) 2006; 45: 636-7.

(C) Tilakaratne et al.; Licensee Bentham Open.

This is an open access article licensed under the terms of the Creative Commons Attribution Non-Commercial License (http://creativecommons.org/licenses/by-nc/3.0/) which permits unrestricted, non-commercial use, distribution and reproduction in any medium, provided the work is properly cited. 